\title{
Evaluation of Facture Criteria Considering Complex Loading Paths in Cobalt Alloy Tube Hydroforming
}

\author{
R. S. Lee ${ }^{1}$, H. Y. Chiu ${ }^{1}$, Y. J. Chen ${ }^{1}$, Y. C. Lo $^{2}$ and C. C. Wang ${ }^{2}$ \\ ${ }^{1}$ Department of Mechanical Engineering, National Cheng Kung University, 701 Taiwan, R. O. China \\ ${ }^{2}$ AeroWin Technology Corporation, 730 Taiwan, R. O. China
}

In this study, a free bulge test simulation was performed with conditions corresponding to experiments for a cobalt-alloy tube hydroforming. A 3D strain measurement scheme was introduced to obtain strain data after free bulge tests, and the measured strain values were used in the evaluation of ductile fracture criteria. Cockcroft ductile fracture criterion, Oyane ductile fracture criterion and the modified Cockcroft criterion were adopted for establishing the material constants by simple tensile test and notched tensile test. Experimental data from free bulge tests were used for fracture prediction with these criteria. The results revealed that for the purpose of tube hydrofoming, the modified Cockcroft criterion with strain paths is more accurate than either the Cockcroft ductile fracture criterion or the Oyane ductile fracture criterion. [doi:10.2320/matertrans.MF201119]

(Received August 18, 2011; Accepted January 12, 2012; Published April 11, 2012)

Keywords: ductile fracture criteria, bulge test, tube hydroforming, strain paths

\section{Introduction}

Due to the unique material properties of aerospace materials and the complex geometry of tubular parts in the aero industry, the hydroforming process has been introduced into the aero manufacturing industry in virtue of better formability with complex geometry. In this study, a cobaltbased HAYNES-188 (HA-188) super alloy was chosen for the tube hydroforming process (THF). In the literatures, there has been a large number of fracture criteria used to predict the occurrence of fractures in the metal formation process. In this paper, the suitability of three criteria for hydroforming applications was investigated.

Many researchers have studied the dominating parameters by which to predict the initiation of fractures in THF process. Ahmed and $\mathrm{Hashmi}^{1)}$ reviewed the developments in bugle formation of tubular components, and they studied the relations among internal pressure, axial loading, and clamping load for the design of dies and tools. Plancak et al. ${ }^{2)}$ presented a new analytical method to determine the coefficient of friction in the hydroforming of tubes, based upon the tube setting test. Xing and Makinouchi ${ }^{3)}$ proposed that the occurrence of a deformed shape in the earlier free forming stage is very important for controlling the final thickness distribution and the components quality, and the inhouse FE program was used to simulate hydroformation in order to control the material flow and to prevent final failure modes from occurring.

When strain paths during the forming process are linear and simple, forming limit diagrams (FLD) are useful. However, in cases with nonlinear strain paths, such as in the THF, internal pressure can form a complex stress state and nonlinear strain paths, and the accuracy of fracture prediction may thus be lowered as a result. Other than using the FLD for prediction, Lei et al. ${ }^{4)}$ used Oyane ductile fracture criterion to predict limit strains and bursting pressure in the hydroforming process. The material constant in the criterion can be determined by using a forming limit diagram from a series of bulge tests. Song ${ }^{5)}$ and his team replaced the
Oyane fracture criterion with the criterion established by Cockcroft and Latham. However, Cockcroft and Latham did not consider the hydrostatic stress term in the criterion.

\section{Theoretical Background and Analysis}

Based on deformation history, the fracture criterion proposed by Cockcroft and Latham ${ }^{6}$ stated that a fracture in a ductile material occurs when the integral of the maximum principal stress over the effective strain reaches a critical value. This criterion can be expressed as:

$$
\int_{0}^{\varepsilon_{\mathrm{f}}} \sigma_{\text {max. }} \mathrm{d} \bar{\varepsilon}=c_{1}
$$

Where $\sigma_{\text {max. }}, \mathrm{d} \bar{\varepsilon}, \varepsilon_{\mathrm{f}}$, and $c_{1}$ denote the maximum principal stress, effective strain increment, effective strain at fracture and ductile fracture criterion constant, respectively.

In 1980, Oyane ${ }^{7)}$ proposed a ductile fracture criterion based on pore-free material that was applied to predict fracture strain and the working limit in forming processes. In this criterion, the assumptions were that the hydrostatic stress affects the occurrence of a ductile fracture, shown as:

$$
\int_{0}^{\varepsilon_{\mathrm{f}}}\left(A+\frac{\sigma_{\mathrm{H}}}{\bar{\sigma}}\right) \mathrm{d} \bar{\varepsilon}=c_{2}
$$

Where $\sigma_{\mathrm{H}}, \bar{\sigma}, \mathrm{d} \bar{\varepsilon}$, and $\varepsilon_{\mathrm{f}}$ denote the hydrostatic stress, effective stress, effective strain increment, and effective strain at fracture, respectively. $A$ and $c_{2}$ are material constants that can be obtained from a simple tensile test and a notched tensile test. In eq. (2), fracture occurs when the value of the left-hand side reaches the critical fracture energy value.

It can be seen that the energy accumulation value at a local area with the fracture criterion proposed by Cockcroft and Latham can be calculated. When the value reaches the material constant, then ductile fracture occurs on a specific point in the local area. However, if the strain state on a specific point exhibits bi-axial tension, the accuracy of prediction will be lost by using Cockcroft ductile criterion. To improve the criterion, Zhuang $^{8)}$ proposed a modified 


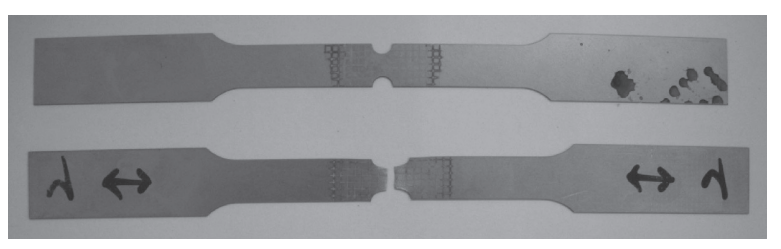

Fig. 1 Specimens for notched tensile test.

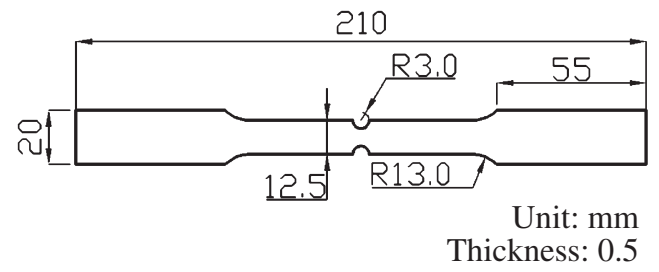

Fig. 2 Dimensions of notched specimen.

criterion based on Cockcroft criterion with strain path effect which was considered by adding strain ratio into the criterion, shown as:

$$
\frac{1}{(1+|\rho|)^{\beta}} \int_{0}^{\varepsilon_{\mathrm{f}}} \sigma_{\text {max. }} \mathrm{d} \bar{\varepsilon}=c_{3}
$$

Where $\sigma_{\text {max. }}, \mathrm{d} \bar{\varepsilon}, \varepsilon_{\mathrm{f}}$, and $\rho$ denote the maximum principal stress, effective strain increment, effective strain at fracture and the strain ratio. $\beta$ and $c_{3}$ are material constants, respectively. In the modified Cockcroft criterion, material constants can be solved by two material tests with different strain paths. From the results of simple tensile test and notched tensile test, the fracture strains with tensioncompression and near plane strain can be obtained. Because of the limitation of the strain measurement technique, the complete grids at the nearest fracture site can be measured as the fracture strains. The integral value is slightly less than the actual fracture, but it is a critical value related to the occurrence of a ductile fracture. When the integral value on a specific point in the local area reaches the critical value, the occurrence of fracture is determined. In this paper, the suitability for hydroforming applications by using the three criteria was evaluated.

\section{Material Tests and Free Bulge Test}

\subsection{Material tests}

The constants of the ductile fracture criteria come from the material tests. Therefore, simple tensile tests and notched tensile tests with notched tensile specimen were performed with grid etching on the surfaces of specimens, and $2 \mathrm{~mm}$ square pattern are used. The specimens and dimensions for notched tensile tests are shown in Figs. 1 and 2. The obtained flow stress and fracture strains were used to determine the energy integral values in criteria.

\subsection{Free bulge test}

In order to obtain the formability data for tube hydroforming processes, free bulge tests were performed and a non-axial-symmetric die set was designed for this test. The tube for the test was prepared from blank sheet with HA-188
Table 1 Chemical composition.

\begin{tabular}{cccccc}
\hline Material & \multicolumn{5}{c}{ Chemical composition (mass\%) } \\
\hline \multirow{2}{*}{ HA-188 } & $\mathrm{Cr}$ & $\mathrm{Ni}$ & $\mathrm{W}$ & $\mathrm{La}$ & $\mathrm{Co}$ \\
\cline { 2 - 6 } & 22 & 22 & 15 & 0.09 & Balanced \\
\hline
\end{tabular}

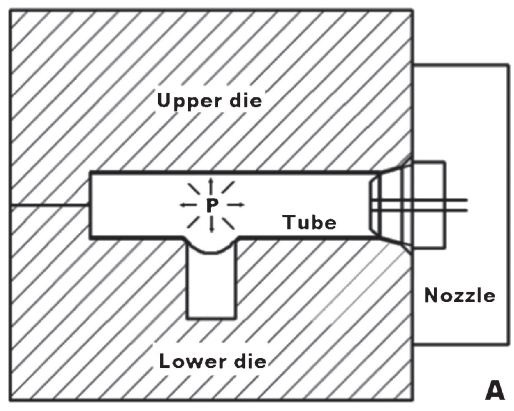

Fig. 3 Sectional drawing of die set.

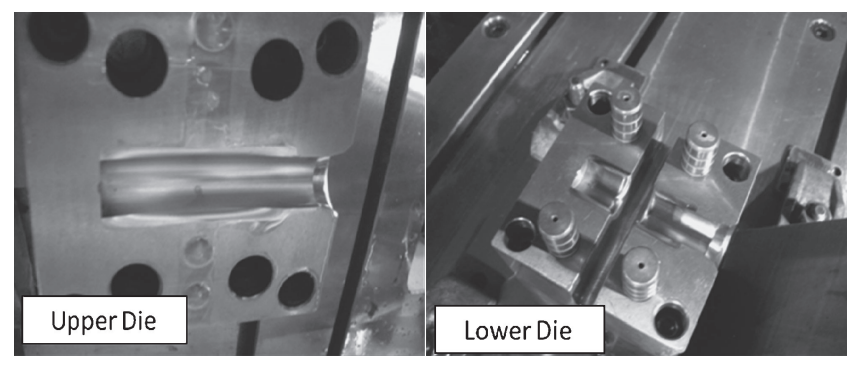

Fig. 4 Die set for the free bulge test.

Table 2 Free bulge test conditions.

\begin{tabular}{cc}
\hline Testing machine & $\begin{array}{c}1000 \text { ton Hydraulic } \\
\text { forming machine }\end{array}$ \\
\hline Tube geometry & $\phi 20 \mathrm{~mm} \times 94 \mathrm{~mm}$ \\
\hline Tube thickness & $0.5 \mathrm{~mm}$ \\
\hline Testing temperature & Room temperature $\left(20^{\circ} \mathrm{C}\right)$ \\
\hline Maximum pressure & $66 \mathrm{MPa}$ \\
\hline
\end{tabular}

alloy through pre-forming, bending, sizing, and welding to form the tubular specimen, and free bulge tests were executed on a hydraulic machine. The chemical composition of HA188 alloy is given in Table 1.

Non-axial-symmetric die is used to prevent fracture occurring on the welding tube site and instead of occurring on the free bulge area. The die designed is shown in Fig. 3. ${ }^{9}$ ) Tip radius of lower die is ten times tube thickness. The size of fillets on both edges of concaves in longitudinal direction, which were designed to prevent stress concentration, is gradual increasing from the left side to the middle of the concave, and then gradual decreasing to the right side. Die set is shown in Fig. 4.

The experimental conditions of free bulge test are listed in Table 2.9) Before free bulge tests were performed, grid etching on tubes was carried out. A tube is put into the concave of lower die, sealed with upper die and nozzle, and 


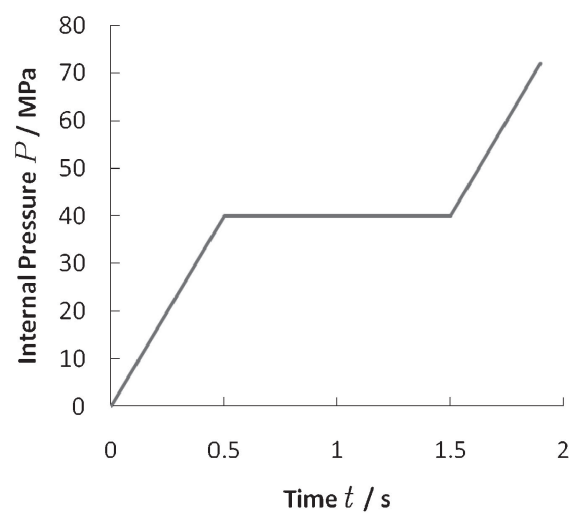

Fig. 5 Loading path in free bulge test.

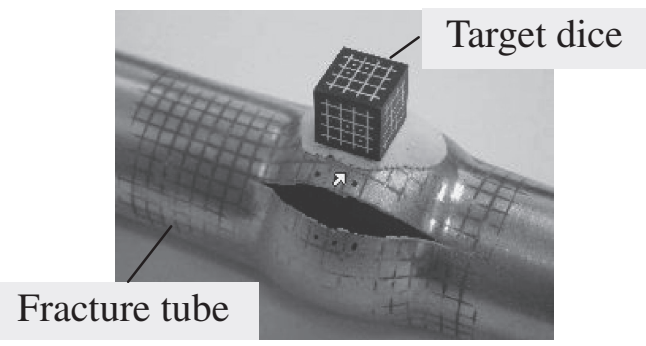

Fig. 6 Set-up for 3D strain measurement.

then oil is pumped into the tube. At the beginning, the pressure was increased according to a loading path as in Fig. 5 and stop until fracture occurs and the maximum pressure was known. And then incremental bulging with lower maximum pressure was preformed. Strains at the highest point of free bulge area were measured through image processing after tests, and used to evaluate the criteria later.

Strains on tubes were measured by a 3D strain measurement scheme. The grids were loaded into a program for strain measurement by photographing. The program calibrates the pictures by using a special target dice as its unit length. Two pictures with different directions are required to locate the position of the dice in the pictures. A set-up picture is shown as Fig. 6. The fracture strain was measured from a grid which was the most closest to fracture site. Thus, fracture predictions from the criteria in this study are critical strains before fracture occurrence.

\section{Evaluation of Ductile Fracture Criteria}

\subsection{Establishment of material constants in ductile fracture criteria}

Once fracture strain values of two material tests are known, material constants in the criteria can be solved with these data. Through image processing with taking pictures for the grids near fracture sites on tensile test specimens, fracture strains were determined so that integral value in Cockcroft criterion was obtained. Notches result different strain paths in the middle of specimens when the specimen under uniaxial tension. Therefore, material constants in Oyane criterion and the modified Cockcroft criterion were solved each by two integral values from simple tensile test and notched tensile test.
Table 3 Details of the material model.

\begin{tabular}{cc}
\hline Flow stress (MPa) & $\sigma=1998.99 \varepsilon^{0.4716}$ \\
\hline Material model & Anisotropic_Elastic_Plastic \\
\hline Element formulation & Belytschko-Lin-Tsay Shell \\
\hline Planar anisotropy & 0.871 \\
\hline
\end{tabular}

Table 4 Simulation conditions.

\begin{tabular}{cc}
\hline Tube geometry & $\phi 20 \mathrm{~mm} \times 94 \mathrm{~mm}$ \\
\hline Tube thickness & $0.5 \mathrm{~mm}$ \\
\hline Mesh count (only tube) & 23751 \\
\hline Coulomb friction coefficient & 0.1 \\
\hline
\end{tabular}

\subsection{FE Simulation conditions}

FE method was applied in this study to calculate deformation and stress distribution of material tests and analyze free bulge test. The commercial software LSDYNA3D was used for simulation in this study. The flow stress of HA-188 described by power law from the simple tensile test was used in all simulations. Belytschko-Lin-Tsay shell element was used for the material model in simulation and the details are shown in Table 3.

3D geometries of dies and a tubular specimen were established as IGES files and used in simulation for free bulge test. The dies were set to be rigid, but only tube undergoes plastic deformation. Also, all boundary conditions in simulation setup were determined according to the test conditions. The simulation conditions are listed in Table 4.

\subsection{Evaluation method}

Through FE simulation, stresses and strains in the deformation history on a specific point in two material tests are obtained from the post-processor and the criterion is determined. With the material constants, $c_{1}, c_{2}$, and $c_{3}$, other fracture strains predicted by the criteria with different strain paths can be solved with FE simulation.

For example, when the integral value on any point with a stain path from simulation results reaches the material constant, $c_{3}$, the integration procedure is stopped, and fracture occurrence is determined. From the definition of the criterion, it can be seen that the final effective strain value on the stopped step in simulation is the fracture strain for the point. And the major strain and minor strain which correspond to this effective strain can be found in the simulation result.

From the above mentioned method, fracture strains with different strain paths can be calculated from simulation results. By plotting these calculated fracture strains on a diagram and connect them to become a curve. This curve is a theoretical forming limit curve instead of experimental curve. By repeating the above method, there were three forming limit curves for the three criteria in this study. These curves were used to predict the free bulge test simulation and compared with experiments. 
Table 5 Result of free bulge test.

\begin{tabular}{ccccc}
\hline Tube & A & B & C & D \\
\hline Maximum effective strain & 0.515 & 0.395 & 0.386 & 0.338 \\
\hline Maximum pressure $(\mathrm{MPa})$ & 66 & 60 & 58 & 56 \\
\hline Bulging result & Fracture & Safe & Safe & Safe \\
\hline
\end{tabular}

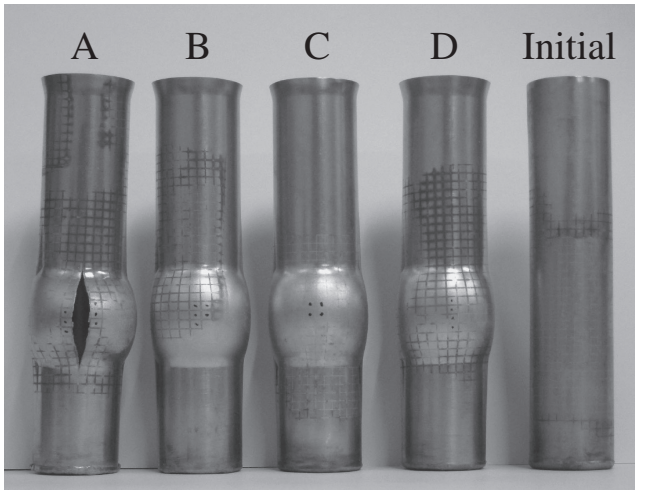

Fig. 7 Specimens of free bulge test.

Table 6 Material constants in the criteria.

\begin{tabular}{cc}
\hline Criterion & Material constants \\
\hline Cockcroft & $c_{1}=590.6$ \\
\hline Oyane & $A=-0.1133, c_{2}=0.1956$ \\
\hline Modified Cockcroft & $\beta=1.595, c_{3}=322.2$ \\
\hline
\end{tabular}

\section{Results and Discussion}

The results from free bulge tests are shown in Table 5. The specimens are shown in Fig. 7, and symbols are corresponded to Table 5. After fracture strains from material test were measured, the criteria were determined. Material constants are shown in Table 6. Forming limit curves from the criteria were drawn, and fracture strains from material tests and free bulge tests also were plotted in Fig. 8. The maximum effective strains were measured on the highest free bulge area. On tube A, the maximum effective strain is also fracture strain.

The criteria were evaluated by forming limit curves, and compared with the fracture strains as shown in Fig. 8. Hollow dots represent safe strains and solid dots are fracture strains. In Fig. 8, fracture strains from two material tests are located on the second quadrant, and strains from free bugle tests are on the first quadrant. Fracture prediction by the forming limit curve from Cockcroft criterion is close to the results from free bulge tests, but fracture does not happen by prediction. Prediction by Oyane criterion is conservative for free bulge test. The modified Cockcroft criterion agrees well with the results from free bulge tests than the others.

Table 7 is the calculation results by the criteria from the free bulge test simulation. Results of fracture prediction by

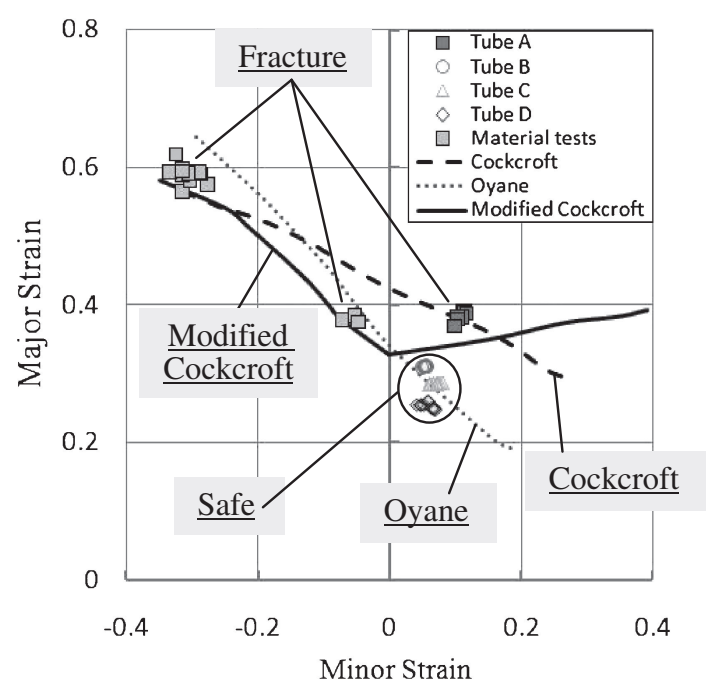

Fig. 8 Fracture prediction on FLD.

Table 7 Comparison of fracture prediction.

\begin{tabular}{ccccc}
\hline Criterion & $\begin{array}{c}\text { Fracture } \\
\text { strain }\end{array}$ & $\begin{array}{c}\text { Critical } \\
\text { value }\end{array}$ & $\begin{array}{c}\text { Integral } \\
\text { result }\end{array}$ & $\begin{array}{c}\text { Error } \\
(\%)\end{array}$ \\
\hline Cockcroft & & 590.6 & 582.7 & -1.34 \\
\cline { 1 - 4 } Oyane & \multirow{2}{*}{0.515} & 0.196 & 0.263 & +34.18 \\
\cline { 1 - 3 } Modified Cockcroft & & 322.2 & 323.1 & +0.28 \\
\hline
\end{tabular}

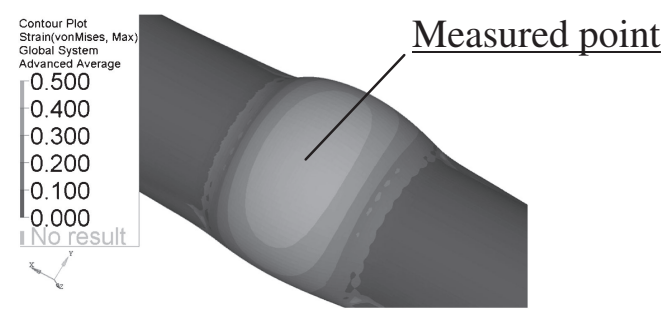

Fig. 9 Measured point from simulation result.

the modified Cockcroft criterion are better than others. Figure 9 shows the measured point from simulation result according to experiments on the highest bulge point. All results revealed that fracture prediction by the modified Cockcroft criterion is more accurate than either Cockcroft or Oyane criterion in free bulge test.

\section{Conclusions}

Forming limit curves have been obtained by Cockcroft, Oyane and the modified Cockcroft criterion with material tests and FE simulation. Free bulge test was performed to obtain fracture strains. The modified Cockcroft criterion shows good agreement with experiment results. The modified Cockcroft criterion has better fracture prediction in tube hydroforming process than original Cockcroft criterion and Oyane criterion. 


\section{Acknowledgement}

The authors would like to gratefully acknowledge National Science Council in Taiwan, R.O.C. and AeroWin Technology Corporation in Taiwan, for financial support through sponsored project NSC 99-2622-E-006-035-CC3 and NSC 100-2622-E-006-035-CC3.

\section{REFERENCES}

1) M. Ahmed and M. S. J. Hashmi: J. Mater. Sci. Technol. 64 (1997) 9-23.
2) M. Plancak, F. Vollertsen and J. Woitschig: J. Mater. Process. Technol. 170 (2005) 220-228.

3) H. L. Xing and A. Makinouchi: Int. J. Mech. Sci. 43 (2001) 1009-1026.

4) L. P. Lei, B. S. Kang and S. J. Kang: J. Mater. Process. Technol. 113 (2001) 673-679.

5) W. J. Song, S. W. Kim, J. Kim and B. S. Kang: J. Mater. Process. Technol. 164-165 (2005) 1618-1623.

6) M. G. Cockcroft and D. J. Latham: J. Inst. Met. 96 (1968) 33-39.

7) M. Oyane, T. Sato, K. Okimoto and S. Shima: J. Mech. Work. Technol. 4 (1980) 65-81.

8) W. L. Zhuang: Master Thesis, Dep. Mech. Eng. NCKU., Taiwan (1990).

9) R. S. Lee, H. Y. Chiu, Y. J. Chen, Y. C. Lo and I. C. Chung: Proc. 5th Int. Conf. on Tube Hydroforming TUBEHYDRO2011, (2011) pp. 183-186. 